\title{
Distinct generation, pharmacology, and distribution of sphingosine 1-phosphate and dihydro-sphingosine 1-phosphate in human neural progenitor cells
}

\author{
Phillip Callihan ${ }^{\mathrm{a}}$, Nicholas C. Zitomer ${ }^{\mathrm{b}}$, Michael V. Stoeling ${ }^{\mathrm{a}, \mathrm{b}}$, Perry C. Kennedy ${ }^{\mathrm{c}}$, Kevin R. \\ Lynch $^{\mathrm{c}}$, Ronald T. Riley ${ }^{\mathrm{b}}$, and Shelley B. Hooks ${ }^{\wedge}$, a \\ aDepartment of Pharmaceutical and Biomedical Sciences, University of Georgia, Athens, GA, \\ USA 30602-2352 \\ bToxicology and Mycotoxin Research Unit, USDA-ARS-SAA, Athens, GA, USA 30604-5677 \\ 'Department of Pharmacology, University of Virginia, Charlottesville, VA, USA 22908
}

\section{Abstract}

In vivo and in vitro studies suggest a crucial role for Sphingosine 1-phosphate (S1P) and its receptors in the development of the nervous system. Dihydrosphingosine 1-phosphate (dhS1P), a reduced form of S1P, is an agonist at S1P receptors, but the pharmacology and physiology of $\mathrm{dhS} 1 \mathrm{P}$ has not been widely studied. The mycotoxin fumonisin $\mathrm{B} 1\left(\mathrm{FB}_{1}\right)$ is a potent inhibitor of ceramide synthases and causes selective accumulation of dihydrosphingosine and dhS1P. Recent studies suggest that maternal exposure to $\mathrm{FB}_{1}$ correlates with the development of neural tube defects (NTDs) in which the neural epithelial progenitor cell layers of the developing brain fail to fuse. We hypothesize that the altered balance of S1P and dhS1P in neural epithelial cells contributes to the developmental effects of $\mathrm{FB}_{1}$. The goal of this work was first to define the effect of $\mathrm{FB}_{1}$ exposure on levels of sphingosine and dh-sphingosine and their receptor active 1phosphate metabolites in human embryonic stem cell-derived neural epithelial progenitor (hESNEP) cells; and second, to define the relative activity of dhS1P and S1P in hES-NEP cells. We found that $\mathrm{dhS} 1 \mathrm{P}$ is a more potent stimulator of inhibition of cAMP and Smad phosphorylation than is S1P in neural progenitors, and this difference in apparent potency may be due, in part, to more persistent presence of extracellular dhS1P applied to human neural progenitors rather than a higher activity at S1P receptors. This study establishes hES-NEP cells as a useful human in vitro model system to study the mechanism of $\mathrm{FB}_{1}$ toxicity and the molecular pharmacology of sphingolipid signaling.

\section{Keywords}

sphingosine 1-phosphate; dihydrosphingosine 1-phosphate; G-protein; coupled receptor; Fumonisin B1; neural progenitor; Smad

\footnotetext{
(C) 2011 Elsevier Ltd. All rights reserved.

`Corresponding author: Shelley B. Hooks, shooks@rx.uga.edu, p: 706-542-2189, f: 706-542-5358, 250 West Green Street, UGA College of Pharmacy, Athens, GA 30602-2352.

Publisher's Disclaimer: This is a PDF file of an unedited manuscript that has been accepted for publication. As a service to our customers we are providing this early version of the manuscript. The manuscript will undergo copyediting, typesetting, and review of the resulting proof before it is published in its final citable form. Please note that during the production process errors may be discovered which could affect the content, and all legal disclaimers that apply to the journal pertain.
} 


\section{INTRODUCTION}

Sphingosine 1-phosphate $\left(\mathrm{S}^{1} \mathrm{P}^{1}\right)$ is a bioactive signaling lipid that is generated in cells from sphingosine by the action of sphingosine kinases (SphKs). S1P activates a family of five Gprotein coupled S1P receptors (S1P 1-5) to regulate diverse physiologic and pathologic processes (Fyrst and Saba, 2010). Dihydrosphingosine, the reduced form that is a biosynthetic precursor of ceramide and thus sphingosine, is also a substrate for SphKs, giving rise to dihydrosphingosine 1-phosphate (dhS1P) (see Fig.1). dhS1P is also a ligand for S1P receptors (Im et al., 2001), but the pharmacology and physiology of dhS1P is less well studied, in part due to its presence at much lower concentrations than S1P in cells.

In vivo and in vitro studies suggest a crucial role for S1P and its receptors in the development of the nervous system (McGiffert et al., 2002; Mizugishi et al., 2005; Pitson and Pebay, 2009). For example, mouse embryos with depleted S1P levels resulting from deletion of $S p h k$ alleles display increased apoptosis and decreased mitosis in neuroepithelial cells of the developing nervous system. In this system, the loss of SphK1/2 activity also resulted in failed neural tube closure in the mouse embryos (Mizugishi et al., 2005). In vitro studies also suggest important effects of S1P signaling in neural stem cells, for example, S1P regulates proliferation of rat neural progenitors (Harada et al., 2004), and we have recently shown that S1P promotes proliferation of human embryonic stem cell-derived neural epithelial progenitor (hES-NEP) cells through a Gi dependent mechanism (Hurst et al., 2008). The role of dhS1P in these effects has not been defined.

Recent studies suggest that maternal exposure to corn crops contaminated with the fungus Fusarium verticilloides that produces the mycotoxin fumonisin $\mathrm{B} 1\left(\mathrm{FB}_{1}\right)$ has been associated with the development of neural tube defects (NTDs) in areas where corn is a dietary staple and diets are likely to be deficient in folate (Marasas et al., 2004; Missmer et al., 2006). $\mathrm{FB}_{1}$ exposure also causes NTDs in a mouse model, in which maternal exposure to $\mathrm{FB}_{1}$ leads to NTD formation in nearly $80 \%$ of pups (Gelineau-van Waes et al., 2005). $\mathrm{FB}_{1}$ is structurally related to sphingoid bases and is a potent inhibitor of ceramide synthase (CS) enzymes, key mediators of de novo sphingolipid biosynthesis (Wang et al., 1991). Thus, $\mathrm{FB}_{1}$ inhibits multiple sphingolipid dependent cellular processes (Merrill et al., 2001; Stevens and Tang, 1997). The sphingoid base analog FTY720 also inhibits CS (Berdyshev et al., 2009) and induces NTDs in a mouse model (Gelineau-van Waes et al., 2008).

Given that FB $_{1}$ or FTY720 exposure and $S p h K$ deletion lead to similar NTDs in mouse models, it has been proposed that the mechanism of $\mathrm{FB}_{1}$ developmental neurotoxicity is related to its effects on sphingolipid metabolism. In de novo sphingolipid biosynthesis, ceramide synthase enzymes are required to convert dihydrosphingosine to dihydroceramide, which is then converted first to ceramide by a desaturase and then to sphingosine by ceramidase (Fig.1). Thus, $\mathrm{FB}_{1}$ inhibition of ceramide synthase typically causes accumulation of dihydrosphingosine, increasing the dh-sphingosine:sphingosine ratio in cells (Enongene et al., 2002; Yoo et al., 1996). Given that both sphingoid bases are substrates for SphKs, $\mathrm{FB}_{1}$ exposure may also alter the pools of S1P and dhS1P. $\mathrm{FB}_{1}$-induced accumulation of S1P and dhS1P has been demonstrated in maternal mouse tissue of pregnant dams (Voss et al., 2009) and dhS1P has been detected in fetal mouse liver from dams fed diets contaminated with $\mathrm{FB}_{1}$ (Riley et al., 2006).

\footnotetext{
${ }^{1} \mathrm{~S} 1 \mathrm{P}$ - sphingosine 1-phosphate; SphK - sphingosine kinases; S1P 1-5 - S1P receptors 1-5; dhS1P - dihydrosphingosine 1phosphate; fumonisin B1 - FB 1 ; neural tube defects - NTDs; ceramide synthase - CS; human embryonic stem cell-derived neural epithelial progenitor - hES-NEP
} 
As described, human epidemiology studies suggest that $\mathrm{FB}_{1}$ may be a developmental neurotoxin causing NTDs, and mouse studies suggest the mechanism may be in part related to disruptions in S1P receptor signaling. However, a human cell system in which mechanistic studies can be carried out is critical to bridge these two systems, given the welldocumented differences between mouse and human progenitor cell signaling pathways (Callihan et al., 2011). We have previously shown that hES-NEP cells express functional $\mathrm{S} 1 \mathrm{P}$ receptors that regulate second messenger production, proliferation, and cellular morphology (Hurst et al., 2008). Therefore, hES-NEP cells represent a potential model system to study the effects of $\mathrm{FB}_{1}$ exposure on $\mathrm{S} 1 \mathrm{P}$ receptor pharmacology and possible mechanisms of NTD development. The twin goals of this work were to define the effect of $\mathrm{FB}_{1}$ exposure on levels of sphingosine and dh-sphingosine and their receptor-active 1phosphate metabolites in hES-NEP cells, and the relative activity of dhS1P and S1P in human neural progenitor cells. We report selective elevation of dhS1P following $\mathrm{FB}_{1}$ exposure and distinct pharmacologic activity and distribution of dhS1P and S1P in hES-NEP cells. Our results suggest that $\mathrm{FB}_{1}$ may specifically induce dhS1P mediated signaling effects in this developmentally important cell type.

\section{METHODS AND MATERIALS}

\subsection{Materials}

Fumonisin B1 (Cayman Chemical), S1P, dhS1P, and C17 S1P (Avanti Polar Lipids), C20sphinganine (Matreva), IBMX and forskolin (Sigma-Aldrich), $\left[{ }^{3} \mathrm{H}\right]$-adenine and $\left[{ }^{3} \mathrm{H}\right]-$ myoinositol (American Radiolabelled Chemicals), $\left[{ }^{14} \mathrm{C}\right]$-cAMP (GE Healthcare), pSmad2 antibody (Cell Signaling Technologies). Activin A was generously provided by Dr. Steven Dalton, University of Georgia. S1P stocks were prepared in $\mathrm{H}_{2} \mathrm{O}$ containing $1 \%$ fatty acidfree BSA, and dhS1P stocks were prepared in DMSO. Both stocks were diluted into culture media containing $0.1 \%$ fatty acid-free BSA. Vehicle controls were included for each experiment.

\section{2 hES-NEP cell culture}

Commercially available stocks of hES-NEP cells derived from WA09 human embryonic stem cells were used (available as STEMEZ ${ }^{\mathrm{TM}} \mathrm{hNP}^{\mathrm{TM}}$, Aruna Biomedical). Tissue culture plates were first coated with matrigel (BD Biosciences) diluted 1:200 in NEUROBASAL ${ }^{\mathrm{TM}}$ medium (GIBCO) for 1.5 hours at room temperature, and washed with HyClone ${ }^{\circledR}$ DPBS/ MODIFIED (1X) with calcium \& magnesium (Thermo Scientific) prior to application of media containing cells. Cells were grown in $\mathrm{AB}^{\mathrm{TM}}$ media with ANS ${ }^{\mathrm{TM}}$ (Aruna Biomedical) supplemented with $2 \mathrm{mM} \mathrm{L-glutamine} \mathrm{(Sigma)} \mathrm{and} \mathrm{20ng/mL} \mathrm{b-FGF} \mathrm{(R \& D} \mathrm{Systems).} \mathrm{Cells}$ were passaged approximately every 48 hours and split 1:2 following manual dissociation using a $25 \mathrm{~cm}$ cell scraper (Sarstedt).

\subsection{Liquid chromatography/tandem mass spectrometry LC-MS}

hES-NEP cells were plated in 6-well plates (BD Falcon) at 300,000 cells/well and allowed to grow to confluency $(\sim 500,000$ cells/well). Cells were treated as described and collected using a $25 \mathrm{~cm}$ cell scraper and pelleted at $200 \times \mathrm{g}$ for $5 \mathrm{~min}$ at $4^{\circ} \mathrm{C}$ in a $15 \mathrm{~mL}$ conical culture tube. Cell pellets and media were separated and stored at $-80^{\circ} \mathrm{C}$ until extraction. Samples were extracted and analyzed using a modification of the method described previously (Zitomer et al., 2008). Briefly, cell pellets were thawed on ice and $1.0 \mathrm{~mL}$ of cell extraction mixture [1:1 acetonitrile:water containing $5 \%$ formic acid and $60 \mathrm{pmol} / \mathrm{ml}$ of $\mathrm{C} 20$ sphinganine and $\mathrm{C} 17$-sphingosine 1-P internal standards] is added to the tube and vortexed gently. Samples were then placed in a sonicator at $50^{\circ} \mathrm{C}$ for $1 \mathrm{hr}$. Following sonication, samples were rocked gently for $2 \mathrm{hrs}$ at room temperature. Extracted cell samples were then centrifuged, and $0.5 \mathrm{~mL}$ of supernatant was clarified using $1.5 \mathrm{~mL} 0.45 \mu \mathrm{m}$ nylon centrifuge 
tube filters (COSTAR ${ }^{\circledR}$, Corning Inc., Corning. NY, USA). Samples were transferred to LC-MS vials and analyzed via LC-MS as described previously (Zitomer et al., 2008). Media samples $(0.5 \mathrm{ml})$ were extracted and analyzed using a similar protocol except that the extraction mixture was $0.5 \mathrm{~mL}$ of acetonitrile containing $10 \%$ formic acid and $120 \mathrm{pmol} / \mathrm{mL}$ of C20 sphinganine and C17-sphingosine 1-P internal standards.

The limits of detection in cells were $0.25 \mathrm{pmol} / 500,000$ cells (dh-sphingosine), $0.625 \mathrm{pmol} /$ 500,000 cells (sphingosine), $0.625 \mathrm{pmol} / 500,000$ cells (dh-sphingosine $1-\mathrm{P}$ ), and 0.625 $\mathrm{pmol} / 500,000$ cells (sphingosine 1-P). The limits of detection in media samples were 0.5 $\mathrm{pmol} / \mathrm{mL}$ (dh-sphingosine), $1.25 \mathrm{pmol} / \mathrm{mL}$ (sphingosine), $1.25 \mathrm{pmol} / \mathrm{mL}$ (dh-sphingosine 1$\mathrm{P}$ ), and $1.25 \mathrm{pmol} / \mathrm{mL}$ (sphingosine 1-P). Data shown are representative of at least 2 experiments performed in duplicate.

\subsection{Generation of S1P receptor expressing $\mathrm{CHO}$ cells}

SIP receptor expressing $\mathrm{CHO}$ cells were generated as described previously (Kennedy et al., 2011). Briefly, CHO-K1 cells were transfected with pcDNA containing DNA sequences for HA-tagged S1P1, 2, 3, 4, or 5. Cells expressing the desired receptor were sorted using antihemagglutinin-phycoerythrin fluorescent antibody (Milentyi Biotec Inc, Auburn, CA) and a FACSVantage SE Turbo Sorter (BD Biosciences, Franklin Lakes, NJ). Cell populations were maintained in F-12K medium supplemented with $10 \%$ fetal bovine serum and $1 \mathrm{mg} / \mathrm{mL}$ geneticin (G418).

\subsection{Adenylyl cyclase activity}

We used a modified version of established protocols (Hettinger-Smith et al., 1996). hES$\mathrm{NEP}$ or CHO-K1 cells were plated in 24-well plates (BD Falcon) and labeled with $0.6 \mu \mathrm{Ci}$ $\left[{ }^{3} \mathrm{H}\right]$-adenine for three hours in the presence or absence of $200 \mathrm{ng} / \mathrm{mL}$ pertussis toxin (US

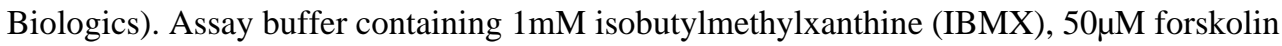
(FSK), and varying concentrations of S1P or dhS1P (Avanti Polar Lipids) were added to the cells for 20 minutes at $37^{\circ} \mathrm{C}$. Reactions were terminated by aspiration followed by addition of stop solution containing $1.3 \mathrm{mM}$ cAMP and $2 \%$ sodium dodecyl sulfate. $\left[{ }^{14} \mathrm{C}\right]$-cAMP stock was added to each well to control for recovery of cAMP, followed by perchloric acid to lyse cells. Lysates were neutralized with $\mathrm{KOH}$ and cAMP was isolated using sequential column chromatography over Dowex AG-50-W4 cationic exchange resin (Bio-Rad, Hercules, CA) followed by neutral alumina columns. The resulting eluate was subjected to scintillation counting after the addition of scintillation cocktail. Data were analyzed using GraphPad Prism software using a nonlinear regression dose-response model. Data shown are representative of at least 3 experiments performed in triplicate. Calculated S1P and dhS1P $\mathrm{EC}_{50}$ values from three independent experiments were compared using a t-test, with p-value less than 0.05 considered significant.

\subsection{Inositol phosphate assay}

Production of inositol phosphates (IP) was measured using established protocols (Hepler et al., 1987). Briefly, hES-NEP or CHO-K1 cells were plated in 24-well plates and grown to $\sim 80 \%$ confluency. Cells were incubated with $1 \mu \mathrm{Ci} /$ well $\left[{ }^{3} \mathrm{H}\right]$ myo-inositol (American Radiolabeled Chemical, St. Louis, MO) for 18 hours to label the cellular pool of phosphatidyl inositol. The cells were treated with varying concentrations of S1P or dhS1P in the presence of $10 \mathrm{mM}$ lithium chloride, to inhibit the degradation of inositol phosphates, for 30 minutes at $37^{\circ} \mathrm{C}$. Following aspiration, cells were lysed in cold $50 \mathrm{mM}$ formic acid and neutralized with ammonium hydroxide. The lysates were loaded onto columns of AG 1-X8 anion exchange resin (BioRad, Hercules, California). The columns were washed with water and dilute ammonium formate to remove unhydrolyzed lipids. The $\left[{ }^{3} \mathrm{H}\right]$ IPs were then eluted with $1.2 \mathrm{M}$ ammonium formate/ $0.1 \mathrm{M}$ formic acid, and added to scintillation cocktail for 
counting. In some experiments, cells were treated with $100 \mathrm{ng} / \mathrm{mL}$ pertussis toxin for 18 hours prior to assay. Data were analyzed using GraphPad Prism software using a nonlinear regression dose-response model. Data shown are representative of at least 3 experiments performed in triplicate. Calculated $\mathrm{S} 1 \mathrm{P}$ and $\mathrm{dhS} 1 \mathrm{P} \mathrm{EC}_{50}$ values from three independent experiments were compared using a t-test, with p-value less than 0.05 considered significant.

\subsection{Smad2 Phosphorylation}

hES-NEP cells were plated in 24 well plates. dhS1P or S1P was added to the cells for 45 minutes at $37^{\circ} \mathrm{C}$. The reaction was terminated by aspirating the media and adding $100 \mu \mathrm{L}$ SDS-PAGE sample buffer. Cells lysates were boiled for 5 minutes in protein sample buffer, separated by SDS-PAGE, transferred to nitrocellulose membranes, and immunoblotted using a primary antibody targeted against phospho-Smad2 (Cell Signaling Technologies, \#3108) and peroxidase conjugate secondary antibody (Bethyl Laboratories, Montgomery, TX). Bands were visualized using SuperSignal Chemiluminescent substrate (Pierce, Rockford, IL). Densitometry analysis was performed using Total Lab 1D Gel Analysis software. Background bands were not subtracted out and all lanes and bandwidths were of equal size. Densitometry results for phospho-Smad2 were normalized to GAPDH to control for loading. Each figure is representative of at least 3 experiments performed in duplicate. Statistical increases in Smad phosphorylation over basal levels were determined using an unpaired, two-tailed T-test using mean and SEM. P-values less than 0.05 were considered significant.

\subsection{GTPYS Binding}

Membrane fractions were prepared from CHO cells stably transfected to over-express the human S1P4 receptor as described (Hooks et al., 2001). These membrane fractions were incubated in 96 well format in $100 \mu \mathrm{L}$ of binding buffer [ $50 \mathrm{mM} \mathrm{N}-2$ -

hydroxyethylpiperazine- $N^{\prime}$-2-ethanesulfonic acid (HEPES), $10 \mathrm{mM} \mathrm{MgCl}_{2}$, and $100 \mathrm{mM}$ $\mathrm{NaCl}$ at $\mathrm{pH} 7.5$ containing $0.1 \%$ fatty acid free bovine serum albumin] with $5 \mu \mathrm{g}$ saponin, $11.5 \mu \mathrm{M}$ GDP, $0.3 \mathrm{nM}\left[\gamma^{-35} \mathrm{~S}\right] \mathrm{GTP}(1200 \mathrm{Ci} / \mathrm{mmol})$ and a concentration range of S1P or $\mathrm{dhS} 1 \mathrm{P}$ for 30 minutes at $30^{\circ} \mathrm{C}$. Membranes were recovered on $\mathrm{GF} / \mathrm{C}$ filters using a 96 well Brandel Cell Harvester (Gaithersburg, MD). Filter plates containing membranes were analyzed for bound radionuclide using a TopCount beta scintillation counter (Packard). Binding data (in Counts Per Minute) were analyzed in GraphPad Prism software using a nonlinear regression sigmoidal dose-response model. Data shown are representative of quadruplicate samples with error bars representing standard error of the mean.

\section{RESULTS}

\subsection{Analysis of sphingolipid metabolites following $\mathrm{FB}_{\mathbf{1}}$}

To determine the effects of $\mathrm{FB}_{1}$ on sphingolipid metabolism in a human neuroepithelial cell, we treated hES-NEP cells with increasing concentrations of $\mathrm{FB}_{1}$ for 48 hours and measured cellular sphingosine and dh-sphingosine levels using LC-MS. We observed a concentrationand time- dependent increase in dh-sphingosine accumulation, with maximal effects observed between $2 \mu \mathrm{M}$ and $20 \mu \mathrm{M} \mathrm{FB}{ }_{1}$ treatment and after 48 hours treatment (Fig.2A, B). Sphingosine levels also increased, but to a much smaller extent than dh-sphingosine levels, and the effect was not consistently observed. We also measured sphingosine and dhsphingosine levels in media, and detected an increase in extracellular dh-sphingosine, but not sphingosine (data not shown).

The accumulation of dh-sphingosine could result in increases in the S1P receptor agonist $\mathrm{dhS} 1 \mathrm{P}$ through the action of sphingosine kinases. Indeed, we observed detectable levels of dhS1P in cells treated with $20 \mu \mathrm{M} \mathrm{FB}_{1}$, but not in untreated control cells (Fig.2C). S1P was not detectable in $\mathrm{FB}_{1}$ treated or untreated cells under these conditions (limit of detection of 
dhS1P and S1P per well: 0.625 pmol; details in Methods.) These data show that 48 hour exposure of hES-NEP cells to $\mathrm{FB}_{1}$ results in accumulation of the sphingoid base dhsphingosine and the bioactive lysophosphosphingolipid dhS1P, but not sphingosine or S1P.

\subsection{Pharmacology of S1P and dhS1P stimulated second messenger production in hES- NEP cells}

The observation that dhS1P, but not $\mathrm{S} 1 \mathrm{P}$, increases after exposure to $\mathrm{FB}_{1}$ raises questions about their relative biological roles. dhS1P is expressed in most biologic systems at much lower levels than $\mathrm{S} 1 \mathrm{P}$ and is an agonist at $\mathrm{S} 1 \mathrm{P}$ receptors, but its activity has not been well defined. Surprisingly, Trojanowska and colleagues have reported markedly different activities of dhS1P and S1P in fibroblasts (Bu et al., 2008; Bu et al., 2006). To define the relative pharmacologic activity of dhS1P and S1P in hES-NEP cells, we determined the potency and efficacy of S1P and dhS1P in two major second messenger pathways downstream of S1P receptors. Inositol triphosphate $\left(\mathrm{IP}_{3}\right)$ is generated by phospholipase $\mathrm{C}$, which is typically activated downstream of activated Gaq, and also by $\mathrm{G} \beta \gamma$ following activation of Gi coupled receptors. cAMP is generated by adenylyl cyclase, which is inhibited by activation of Gai. Given that all S1P receptors couple to either Gi or Gq (or both), the use of these two assays allows complete characterization of endogenous S1P receptor activation.

There was no significant difference in the potency or efficacy of S1P and dhS1P stimulation of phospholipase $\mathrm{C}$ activity in hES-NEP cells, as measured by $\mathrm{IP}_{3}$ accumulation (Fig.3A). Both lipids resulted in robust accumulation of $\mathrm{IP}_{3}$, with an $\mathrm{EC}_{50}$ of approximately $200 \mathrm{nM}$. However, in cAMP accumulation assays, dhS1P was consistently and significantly more potent than S1P at inhibiting adenylyl cyclase activity ( $\mathrm{pEC}_{50} \mathrm{~S} 1 \mathrm{P}: 6.9, \mathrm{pEC}_{50} \mathrm{dhS1P}: 8.15$, $\mathrm{p}=0.045$ ) (Fig.3B). Thus, not only is dhS1P accumulation much higher than S1P accumulation following $\mathrm{FB}_{1}$ exposure, dhS1P also has greater apparent potency in $\mathrm{Gi}$ coupled signaling than S1P in hES-NEP cells.

\subsection{Pharmacology of S1P and dhS1P in Smad phosphorylation}

Trojanowska and colleagues reported that in dermal fibroblasts, dhS1P and S1P showed opposing effects on transforming growth factor- $\beta$ (TGF $\beta$ )/Smad signaling. In these cells, S1P activated Smad phosphorylation while dhS1P inhibited TGF $\beta$ induced Smad phosphorylation (Bu et al., 2008). Smads are key regulators of differentiation and pluripotency pathways in stem cells. If dhS1P and S1P have opposing effects on Smad signaling in neural progenitor cells as reported in fibroblasts, then the selective upregulation of dhS1P versus S1P following $\mathrm{FB}_{1}$ exposure would strongly inhibit Smad activity and tightly control proliferation and differentiation of these cells. However, in hES-NEP cells, we found that both S1P and dhS1P induced Smad2 phosphorylation (Fig. 4A), and neither sphingolipid inhibited activation of Smad2 by the TGF $\beta$ ligand, activin (Fig. 4B). Thus, $\mathrm{dhS1P}$ and S1P do not show opposing effects on Smad signaling in hES-NEP cells. We did observe differences in activity of dhS1P and S1P. dhS1P resulted in higher levels of Smad activity at $1 \mu \mathrm{M}$ and $10 \mu \mathrm{M}$ than equal concentrations of S1P, and the effect of dhS1P was significant at both concentrations, while the effect of S1P was only statistically significant at $10 \mu \mathrm{M}$ (Fig. 4A). These data suggest that dhS1P is more efficacious than S1P at Smad2 phosphorylation in hES-NEP cells, and these lipids regulate Smad signaling though different mechanisms or pathways in neural progenitors versus fibroblasts.

\subsection{Pharmacology of S1P and dhS1P stimulated signaling in stable S1P receptor expressing $\mathrm{CHO}-\mathrm{K} 1$ cell lines}

Our results in hES-NEP cells document that dhS1P is more potent than S1P at a subset of $\mathrm{S} 1 \mathrm{P} / \mathrm{dhS} 1 \mathrm{P}$ stimulated responses, suggesting that dhS1P may have higher activity at one or 
more of the S1P receptors expressed in hES-NEP cells. We have previously reported that hES-NEP cells express S1P receptors 1-3, 5 (Hurst et al., 2008). Further, we have previously shown that dhS1P can activate S1P receptors in a broken cell assay with overexpressed Gi heterotrimers (Im et al., 2001). To determine the relative activity of dhS1P and S1P at each of the S1P receptors in an intact cell system with endogenous G-proteins, we generated stable $\mathrm{CHO}-\mathrm{K} 1$ cell lines expressing individual human S1P receptors. The potency of both ligands was assessed in $\mathrm{IP}_{3}$ and cAMP assays in parental cells and each receptor-expressing cell line.

No S1P-stimulated or dhS1P-stimulated activation of $\mathrm{IP}_{3}$ accumulation or inhibition of cAMP accumulation was observed in the parental CHO-K1 cell line (Figure 5a). Due to distinct G-protein and effector system coupling of the different receptors, some receptor expressing cell lines were able to activate only one of the pathways, for example cells expressing S1P1 showed potent Gi-coupled inhibition of cAMP accumulation but not $\mathrm{IP}_{3}$ accumulation in response to S1P, while S1P2 expressing cells showed no cAMP response, but robust $\mathrm{IP}_{3}$ accumulation in response to $\mathrm{S} 1 \mathrm{P}$.

dhS1P and S1P exhibited similar potencies at S1P receptors S1P1 (cAMP assay), S1P3 (cAMP and $\mathrm{IP}_{3}$ assay), and S1P5 (cAMP). However, dhS1P was significantly less potent than $\mathrm{S} 1 \mathrm{P}$ at receptor $\mathrm{S} 1 \mathrm{P} 2$ ( $\mathrm{IP}_{3}$ assay, Fig.5c). Even though S1P4 is expressed at very low to undetectable levels in hES-NEP cells, we also compared the potency of dhS1P and S1P at S1P4 since the relative activity of dhS1P and S1P has not been defined at this receptor subtype. As observed at SIP2, dhS1P was markedly less potent than S1P at S1P4 receptors in a GTP $\gamma \mathrm{S}$ binding assay (Fig.5d). Thus, dhS1P did not show significantly higher pharmacologic activity at any of the individual S1P receptors, suggesting that receptor selectivity does not account for the increased potency of dhS1P in cAMP inhibition or Smad phosphorylation in hES-NEP cells.

\subsection{Clearance of extracellular S1P and dhS1P in hES-NEP cell cultures}

Given that receptor selectivity does not account for the observed differences in activity, we next predicted that S1P and dhS1P have different half-lives in extracellular media of hESNEP cells to account for the difference in their apparent potency in these cells. To test this hypothesis, we treated hES-NEP cell cultures with $1 \mu \mathrm{M} \mathrm{S1P}$ or dhS1P in complete media for $2,5,10$ and 30 minutes at $37^{\circ} \mathrm{C}$, consistent with the conditions and duration of the cAMP, $\mathrm{IP}_{3}$, and Smad phosphorylation assays. We then isolated the media and cell monolayers, and performed lipid extraction and LC-MS as above to quantify the percent of extracellularly applied dhS1P and S1P in the cellular and media compartments at the end of each incubation period. We found that a significantly higher percent of the original level of dhS1P remained in the media during this time course. At 10 minutes, $70 \%$ of the original $\mathrm{dhS} 1 \mathrm{P}$ remained in media, while only $48 \%$ of S1P remained (Fig. 6). Under these conditions, endogenous levels of cellular S1P and dhS1P were below the limit of detection. However, following addition of extracellular lipid, cell-associated S1P or dhS1P was detected at each time point. Inverse to the trend observed in media, cellular dhS1P levels were significantly lower than S1P levels in cells. dhS1P uptake peaks after 2 minutes and does not exceed $25 \%$ of exogenously applied lipid, while S1P levels continue to increase until 5 minutes and reach levels as much as $80 \%$ of exogenously applied lipid.

\section{DISCUSSION}

Numerous studies have demonstrated an important role for sphingolipid mediators in the regulation of neural development and in the developmental neural toxicity of $\mathrm{FB}_{1}$ exposure in mice, but a human model system to study the mechanism of $\mathrm{FB}_{1}$ toxicity has been lacking. This study was initiated to define the ability of human neuroepithelial progenitor 
cells to generate sphingolipid mediators in response to $\mathrm{FB}_{1}$ exposure and to respond to extracellular S1P and dhS1P. Our findings indicate that hES-NEP cells generate dihydrosphingosine and dhS1P in response to $\mathrm{FB}_{1}$ exposure, but not sphingosine and $\mathrm{S} 1 \mathrm{P}$. Further, we document that dhS1P is a more potent stimulator of inhibition of cAMP and Smad phosphorylation than is S1P in neural progenitors, and this difference in apparent potency may be due, in part, to more persistent presence of extracellular dhS1P applied to human neural progenitors rather than a higher activity at S1P receptors.

This study establishes hES-NEP cells as a powerful human in vitro model system to study the mechanism of $\mathrm{FB}_{1}$ toxicity and the molecular pharmacology of sphingolipid signaling cascades. Multiple studies have established a clear role for phosphosphingolipids in the regulation of many neural cell types; however, results from these studies vary because of the range of model systems differing in species of origin, stage of development, and origin/ derivation of cell lines. There are multiple differences in the regulation of differentiation and pluripotency signaling pathways between human and rodent stem cells, making results from rodent systems difficult to apply to human developmental toxicity. hES-NEP cells are advantageous because they are human cells that grow as an adherent, homogeneous monolayer and can be further differentiated to neurons and glia in culture. Additionally, hES-NEP cells are a stable, renewable in vitro model of the neuroepithelial cell layer responsible for neural tube closure, a critical step in nervous system development.

Closure of the neural tube occurs when neuroepithelial progenitor cells undergo rapid proliferation and migration resulting in the fusion of ectodermal folds at the embryonic midline (Copp and Greene, 2010). Disruption of this process leads to neural tube defects (NTDs) such as spina bifida and anencephaly. NTDs are among the most common birth defects worldwide and their causes are not well understood. The incidence of NTDs is often high in populations where corn is a dietary staple and the diets are likely to be deficient in folic acid and other B vitamins (Marasas et al., 2004). In many of these areas the corn is frequently contaminated with high levels of fumonisin $\mathrm{FB}_{1}$ (Torres et al., 2007) which has been shown to disrupt sphingolipid metabolism and induce NTD in mouse models (Gelineau-van Waes et al., 2009).

$\mathrm{FB}_{1}$ inhibits ceramide synthase, thereby disrupting de novo sphingolipid biosynthesis (Fig. 1). In most systems, this inhibition results in the rapid accumulation of dh-sphingosine, rather than sphingosine (Riley et al., 2001). In hES-NEP cells, endogenous cellular levels of sphingosine are approximately two-fold higher than sphinganine levels (endogenous S1P and dhS1P were below detection limits under the conditions used in our experiments.) Upon $\mathrm{FB}_{1}$ exposure, dh-sphingosine levels increase sharply, with no significant increase in sphingosine, resulting in markedly higher cellular levels of dh-sphingosine compared to sphingosine. Our results also show that $\mathrm{FB}_{1}$ exposure causes increases in cellular levels of dhS1P, but not S1P. However, the magnitude of the increase in dhS1P in hES-NEP cells is much lower than in other cell types and tissues such as mouse red blood cells, liver, kidney and placenta (Gelineau van Waes, personal communication). Indeed, conditioned media removed from $\mathrm{FB}_{1}$ treated $\mathrm{hES}-\mathrm{NEP}$ cells did not contain sufficient phosphosphingolipids to activate S1P receptors in CHO-K1 cells expressing the S1P1 receptor (data not shown), and both S1P and dhS1P were below the detection limit by LC-MS in media samples before or after $\mathrm{FB}_{1}$ exposure. The low level of dhS1P production in $\mathrm{hES}$-NEP cells is consistent with observations in pregnant mice, in which the embryo itself has only modest increases in dhS1P following $\mathrm{FB}_{1}$ exposure, while maternal tissues and blood experience greater increases ((Voss et al., 2009) and unpublished data).

The relatively low level of dhS1P production by hES-NEP cells may be due to cell-specific levels of kinase, lyase, or phosphatase activities. hES-NEP cells apparently express 
sphingosine kinase activity, as the sphingosine analog FTY720 and dihydro-sphingosine are converted to FTY720-P and dhS1P, respectively (data not shown). FTY720 is primarily phosphorylated by SphK2, and both sphingosine and dh-sphingosine are higher affinity substrates for SphK2 than is FTY720. Thus, there is likely sufficient SphK2 activity to generate S1P and dhS1P from the sphingoid bases. Further, the relatively low magnitude of dhS1P accumulation by neural progenitors in response to $\mathrm{FB}_{1}$ may reflect rapid metabolism of the 1-phosphates by lipid phosphate phosphatase activity or lyase activity. Regardless, if $\mathrm{FB}_{1}$ induced increases in dhS1P activity in neural progenitor cells are contributing to the developmental toxicity associated with this toxin, it is likely that the dhS1P derived from maternal tissue and distributed to the embryo by the maternal blood could also serve as a source of extracellular dhS1P.

We also explored the relative ability of extracellular dhS1P and S1P to stimulate receptor signaling cascades in hES-NEP cells. As described above, Bu et al. reported that dhS1P and S1P differentially regulate Smad phosphorylation (Bu et al., 2008). Smad signaling pathways are critical determinants of pluripotency and differentiation in stem cells, and therefore may be relevant in regulating hES-NEP cell biology and regulating neural tube closure. Our results indicate that dhS1P was more active than S1P in activating Smad phosphorylation in hES-NEP cells. This result suggests that dhS1P regulation of Smad2 occurs through different mechanisms in human neural progenitors and fibroblasts. We further found that dhS1P is significantly more potent than S1P in inhibiting adenylyl cyclase activity, while the two lipids had equal potency in activating phospholipase C. As described above, S1P is an important regulator of neural progenitor function, and loss of S1P (and $\mathrm{dhS1P}$ ) synthesis via deletion of the sphingosine kinase enzymes leads to neural tube defects in mice \{Mizugishi, $2005 \# 3430$ \}. This is seemingly at odds with the observation that $\mathrm{FB}_{1}$, which stimulates accumulation of a potent $\mathrm{S} 1 \mathrm{P}$ receptor agonist, also causes neural tube defects in mice. It is likely that in vivo, perturbations of agonist concentrations trigger complex feedback mechanisms to regulate the strength of receptor signaling. Indeed, FTY720-P is a high potency agonist of S1P receptors in vitro but is a functional antagonist in vivo due to its ability to induce receptor internalization and down regulation. Additional studies are required to reconcile these in vitro and in vivo observations.

Given the higher potency and/or activity of dhS1P versus S1P at specific responses in hESNEP cells, we predicted that dhS1P would have higher potency than S1P at one or more of the S1P receptors. However, the above data indicate that dhS1P potency is equal to or less than that of S1P at each individual S1P receptor. Importantly, receptor expression is likely much higher in the transfected CHO cells than hES-NEP cells. Such receptor reserve can inflate the apparent potency of drugs, as reflected in the lower $\mathrm{EC}_{50}$ values of both ligands in cAMP inhibition assays performed in CHO cells compared to hES-NEP cells.

Regardless, , the results from these studies did not explain our findings in hES-NEP cells. A possible trivial explanation for the observed differences in activity of dhS1P and S1P is that, due to differences in solubility, dhS1P and S1P stock solutions were prepared differently. However, the same stocks were used throughout the study, so that if differences in stock preparations affected potency, the difference in potency would be consistent in all assays. In contrast, we observed equal potency in some assays, greater $\mathrm{dhS1P}$ potency in some assays, and greater S1P potency in some assays. Thus, stock preparation does not account for the observed differences.

We next hypothesized that differences in dhS1P vs. S1P metabolism and cellular uptake could account for the signaling differences in hES-NEPs. We found that significantly more $\mathrm{dhS} 1 \mathrm{P}$ remains intact in the extracellular media during the time course of the signaling assays than S1P. Mirroring this trend, we found much lower accumulation of extracellularly applied dhS1P in the cellular fraction than S1P. This prolonged presence of dhS1P in 
mediamay contribute to its higher activity in cAMP inhibition and Smad phosphorylation. However, it should be noted that the fold difference in concentrations of S1P and dhS1P in media is less than the difference in their potency, suggesting other factors also contribute to the higher apparent potency of dhS1P in NEP cells. For example, dhS1P and S1P may have different intrinsic efficacies, which may not have been observed in $\mathrm{CHO}$ cells due to receptor reserve. Finally, the apparent equal potency in $\mathrm{IP}_{3}$ assays may reflect that this effect is likely mediated by the Gq coupled S1P2 receptors, which exhibit a higher potency with $\mathrm{S} 1 \mathrm{P}$ than dhS1P, thus cancelling out the effect of the prolonged presence of dhS1P in media.

The higher accumulation of cellular S1P than dhS1P suggests that cellular uptake indeed contributes to the loss of S1P in the media, but we cannot discount the potential role of differential rates of metabolism by enzymes such as the LPP ecto-phosphatases. It is tempting to speculate that dhS1P is degraded more rapidly than $\mathrm{S} 1 \mathrm{P}$, given that the combined extracellular and cellular S1P remaining after 30 minutes is considerably higher than dhS1P; however, matrix effects between media and cell fraction samples may be quite different, so quantitative comparisons between LC-MS analysis of cellular and media samples are not supported. Regardless, it is clear that within media samples, dhS1P is present at higher concentrations at later time points, and in cellular samples, S1P concentrations are much higher than dhS1P. The sub-cellular localization of sphingolipid metabolizing enzymes and sphingolipid transporters has been the focus of recent studies. Specifically, the movement of sphingoid base 1-phosphates across cell membranes may be mediated by either ABC transporters (ABCC1 or ABCG2) (Takabe et al., 2010) and/or SPNS2 (Hisano et al., 2010). The relative expression, activity, and selectivity of these transporters for dhS1P and S1P in neural progenitors need to be defined to determine their significance in our observations.

In conclusion, we have shown that exposure of hES-NEP cells to $\mathrm{FB}_{1}$ results in preferential increases in dh-sphingosine over sphingosine. In turn, higher levels of the receptor active $\mathrm{dhS} 1 \mathrm{P}$ are produced than S1P. In hES-NEP cells dhS1P is more potent than S1P in stimulating adenylyl cyclase inhibition and Smad phosphorylation assays. These effects do not result from differences in potency at individual S1P receptors, rather from differences in cellular uptake and metabolism. Ultimately, this study indicates that dhS1P may play a role in regulating the response of neural progenitor cells to $\mathrm{FB}_{1}$ exposure.

\section{Acknowledgments}

The authors would like to thank Dr. Janee Gelineau-van Waes for insights throughout the project and Dr. Steven Dalton for providing Activin A. Grant Support: Grant support for PCK: NIH/NIGMS T32 GM008715, KRL: NIH/ NIGMS R01 GM067958, SBH: UGA Research Foundation.

\section{References}

Berdyshev EV, Gorshkova I, Skobeleva A, Bittman R, Lu X, Dudek SM, Mirzapoiazova T, Garcia JG, Natarajan V. FTY720 inhibits ceramide synthases and up-regulates dihydrosphingosine 1-phosphate formation in human lung endothelial cells. The Journal Of Biological Chemistry. 2009; 284:54675477. [PubMed: 19119142]

Bu S, Kapanadze B, Hsu T, Trojanowska M. Opposite effects of dihydrosphingosine 1-phosphate and sphingosine 1-phosphate on transforming growth factor-beta/Smad signaling are mediated through the PTEN/PPM1A-dependent pathway. J Biol Chem. 2008; 283:19593-19602. [PubMed: 18482992]

Bu S, Yamanaka M, Pei H, Bielawska A, Bielawski J, Hannun YA, Obeid L, Trojanowska M. Dihydrosphingosine 1-phosphate stimulates MMP1 gene expression via activation of ERK1/2-Ets1 pathway in human fibroblasts. FASEB J. 2006; 20:184-186. [PubMed: 16278291] 
Callihan P, Mumaw J, Machacek DW, Stice SL, Hooks SB. Regulation of stem cell pluripotency and differentiation by G protein coupled receptors. Pharmacol Ther. 2011; 129:290-306. [PubMed: 21073897]

Copp AJ, Greene ND. Genetics and development of neural tube defects. J Pathol. 2010; 220:217-230. [PubMed: 19918803]

Enongene EN, Sharma RP, Bhandari N, Miller JD, Meredith FI, Voss KA, Riley RT. Persistence and reversibility of the elevation in free sphingoid bases induced by fumonisin inhibition of ceramide synthase. Toxicol Sci. 2002; 67:173-181. [PubMed: 12011476]

Fyrst H, Saba JD. An update on sphingosine-1-phosphate and other sphingolipid mediators. Nat Chem Biol. 2010; 6:489-497. [PubMed: 20559316]

Gelineau-van Waes J, Maddox J, Wilberding J, Voss KA, Riley RT. Placental and Neural Tube Defects After Maternal Fumonisin or FTY720 Exposure: Role of Bioactive Sphingolipids. BirthDefects-Research. 2008; 82:308.

Gelineau-van Waes J, Starr L, Maddox J, Aleman F, Voss KA, Wilberding J, Riley RT. Maternal fumonisin exposure and risk for neural tube defects: mechanisms in an in vivo mouse model. Birth Defects Res A Clin Mol Teratol. 2005; 73:487-497. [PubMed: 15959874]

Gelineau-van Waes J, Voss KA, Stevens VL, Speer MC, Riley RT. Maternal fumonisin exposure as a risk factor for neural tube defects. Adv Food Nutr Res. 2009; 56:145-181. [PubMed: 19389609]

Harada J, Foley M, Moskowitz MA, Waeber C. Sphingosine-1-phosphate induces proliferation and morphological changes of neural progenitor cells. J Neurochem. 2004; 88:1026-1039. [PubMed: 14756825]

Hepler JR, Nakahata N, Lovenberg TW, DiGuiseppi J, Herman B, Earp HS, Harden TK. Epidermal growth factor stimulates the rapid accumulation of inositol $(1,4,5)$-trisphosphate and a rise in cytosolic calcium mobilized from intracellular stores in A431 cells. J Biol Chem. 1987; 262:29512956. [PubMed: 3102480]

Hettinger-Smith BD, Leid M, Murray TF. Chronic exposure to adenosine receptor agonists and antagonists reciprocally regulates the A1 adenosine receptor-adenylyl cyclase system in cerebellar granule cells. J Neurochem. 1996; 67:1921-1930. [PubMed: 8863496]

Hisano Y, Kobayashi N, Kawahara A, Yamaguchi A, Nishi T. The sphingosine 1-phosphate transporter, SPNS2, functions as a transporter of the phosphorylated form of the immunomodulating agent FTY720. J Biol Chem. 2010

Hooks SB, Santos WL, Im DS, Heise CE, Macdonald TL, Lynch KR. Lysophosphatidic acid-induced mitogenesis is regulated by lipid phosphate phosphatases and is Edg-receptor independent. The Journal Of Biological Chemistry. 2001; 276:4611-4621. [PubMed: 11042183]

Hurst JH, Mumaw J, Machacek DW, Sturkie C, Callihan P, Stice SL, Hooks SB. Human neural progenitors express functional lysophospholipid receptors that regulate cell growth and morphology. BMC Neurosci. 2008; 9:118. [PubMed: 19077254]

Im DS, Clemens J, Macdonald TL, Lynch KR. Characterization of the human and mouse sphingosine 1-phosphate receptor, S1P5 (Edg-8) structure-activity relationship of sphingosine1-phosphate receptors. Biochemistry. 2001; 40:14053-14060. [PubMed: 11705398]

Kennedy PC, Zhu R, Huang T, Tomsig JL, Mathews TP, David M, Peyruchaud O, Macdonald TL, Lynch KR. Characterization of a sphingosine 1-phosphate receptor antagonist prodrug. J Pharmacol Exp Ther. 2011; 338:879-889. [PubMed: 21632869]

Marasas WF, Riley RT, Hendricks KA, Stevens VL, Sadler TW, Gelineau-van Waes J, Missmer SA, Cabrera J, Torres O, Gelderblom WC, Allegood J, Martinez C, Maddox J, Miller JD, Starr L, Sullards MC, Roman AV, Voss KA, Wang E, Merrill AH Jr. Fumonisins disrupt sphingolipid metabolism, folate transport, and neural tube development in embryo culture and in vivo: a potential risk factor for human neural tube defects among populations consuming fumonisincontaminated maize. J Nutr. 2004; 134:711-716. [PubMed: 15051815]

McGiffert C, Contos JJ, Friedman B, Chun J. Embryonic brain expression analysis of lysophospholipid receptor genes suggests roles for $\mathrm{s} 1 \mathrm{p}(1)$ in neurogenesis and $\mathrm{s} 1 \mathrm{p}(1-3)$ in angiogenesis. FEBS Lett. 2002; 531:103-108. [PubMed: 12401212] 
Merrill AH Jr, Sullards MC, Wang E, Voss KA, Riley RT. Sphingolipid metabolism: roles in signal transduction and disruption by fumonisins. Environ Health Perspect. 2001; 109(Suppl 2):283-289. [PubMed: 11359697]

Missmer SA, Suarez L, Felkner M, Wang E, Merrill AH Jr, Rothman KJ, Hendricks KA. Exposure to fumonisins and the occurrence of neural tube defects along the Texas-Mexico border. Environ Health Perspect. 2006; 114:237-241. [PubMed: 16451860]

Mizugishi K, Yamashita T, Olivera A, Miller GF, Spiegel S, Proia RL. Essential role for sphingosine kinases in neural and vascular development. Mol Cell Biol. 2005; 25:11113-11121. [PubMed: 16314531]

Pitson SM, Pebay A. Regulation of stem cell pluripotency and neural differentiation by lysophospholipids. Neurosignals. 2009; 17:242-254. [PubMed: 19816061]

Riley RT, Enongene E, Voss KA, Norred WP, Meredith FI, Sharma RP, Spitsbergen J, Williams DE, Carlson DB, Merrill AH Jr. Sphingolipid perturbations as mechanisms for fumonisin carcinogenesis. Environ Health Perspect. 2001; 109(Suppl 2):301-308. [PubMed: 11359699]

Riley, RT.; Voss, KA.; Speer, M.; Stevens, VL.; Waes, JG-v. Fumonisin inhibition of ceramide synthase: A possible risk factor for human neural tube defects. In: Hirabayashi, Y.; Merrill, A.; Igarashi, Y., editors. Sphingolipid Biology. Toyko: Springer Verlag; 2006. p. 345-361.

Stevens VL, Tang J. Fumonisin B1-induced sphingolipid depletion inhibits vitamin uptake via the glycosylphosphatidylinositol-anchored folate receptor. J Biol Chem. 1997; 272:18020-18025. [PubMed: 9218430]

Takabe K, Kim RH, Allegood JC, Mitra P, Ramachandran S, Nagahashi M, Harikumar KB, Hait NC, Milstien S, Spiegel S. Estradiol induces export of sphingosine 1-phosphate from breast cancer cells via ABCC1 and ABCG2. J Biol Chem. 2010; 285:10477-10486. [PubMed: 20110355]

Torres OA, Palencia E, Lopez de Pratdesaba L, Grajeda R, Fuentes M, Speer MC, Merrill AH Jr, O'Donnell K, Bacon CW, Glenn AE, Riley RT. Estimated fumonisin exposure in Guatemala is greatest in consumers of lowland maize. J Nutr. 2007; 137:2723-2729. [PubMed: 18029490]

Voss KA, Riley RT, Snook ME, Waes JG. Reproductive and sphingolipid metabolic effects of fumonisin $\mathrm{B}(1)$ and its alkaline hydrolysis product in $\mathrm{LM} / \mathrm{Bc}$ mice: hydrolyzed fumonisin $\mathrm{B}(1)$ did not cause neural tube defects. Toxicol Sci. 2009; 112:459-467. [PubMed: 19783636]

Wang E, Norred WP, Bacon CW, Riley RT, Merrill AH Jr. Inhibition of sphingolipid biosynthesis by fumonisins. Implications for diseases associated with Fusarium moniliforme. J Biol Chem. 1991; 266:14486-14490. [PubMed: 1860857]

Yoo HS, Norred WP, Showker J, Riley RT. Elevated sphingoid bases and complex sphingolipid depletion as contributing factors in fumonisin-induced cytotoxicity. Toxicol Appl Pharmacol. 1996; 138:211-218. [PubMed: 8658522]

Zitomer NC, Glenn AE, Bacon CW, Riley RT. A single extraction method for the analysis by liquid chromatography/tandem mass spectrometry of fumonisins and biomarkers of disrupted sphingolipid metabolism in tissues of maize seedlings. Anal Bioanal Chem. 2008; 391:2257-2263. [PubMed: 18488202] 


\section{Highlights}

- Exposure of human neural progenitors to the toxin FB1 increases dhsphingosine levels

- S1P receptor-active dhS1P also accumulates, increasing the dhS1P:S1P ratio

- dhS1P is more potent than S1P for Smad activation and cAMP inhibition in progenitors

- These distinctions reflect differences in cellular uptake, not receptor selectivity

- dhS1P signaling may contribute to neural progenitor cells' response to FB1 exposure 


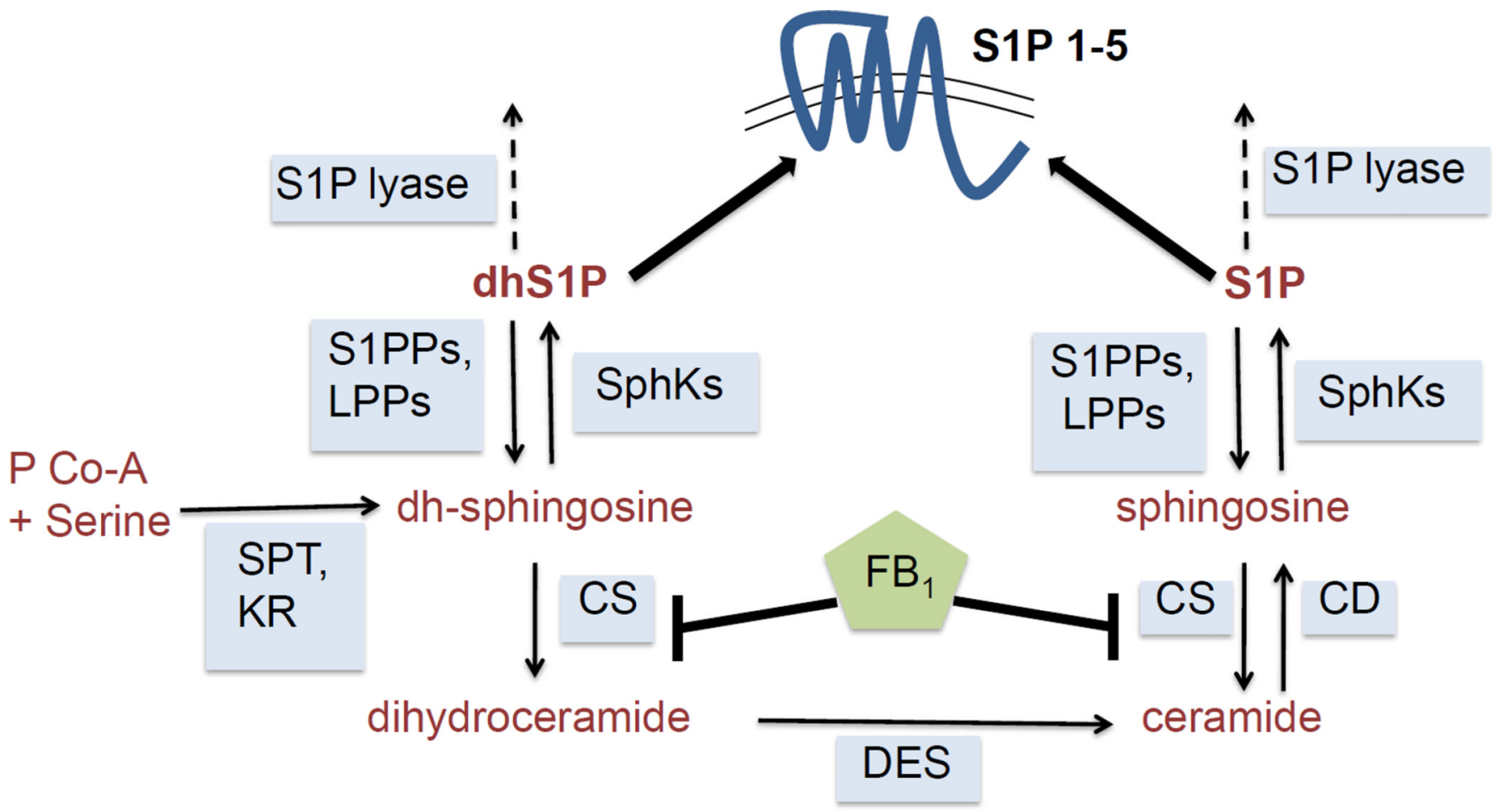

Figure 1. Model of sphingolipid metabolism

This schematic depicts the de novo and salvage pathways of sphingolipid metabolism. Sphingosine 1-phosphate phosphatases (S1PPs), lipid phosphate phosphatases (LPPs), sphingosine kinases (SphKs), S1P receptors 1-5 (S1P 1-5), serine palmitoyl transferase (SPT), ketosphingosine reductase (KR), ceramide synthase (CS), ceramidase (CD), sphingosine 1-phosphate (S1P), dihydrosphingosine 1-phosphate (dhS1P), dihydroceramide desaturase (DES), Fumonisin B1 (FB 1 ), Palmitoyl Co-A (P Co-A). 
A.

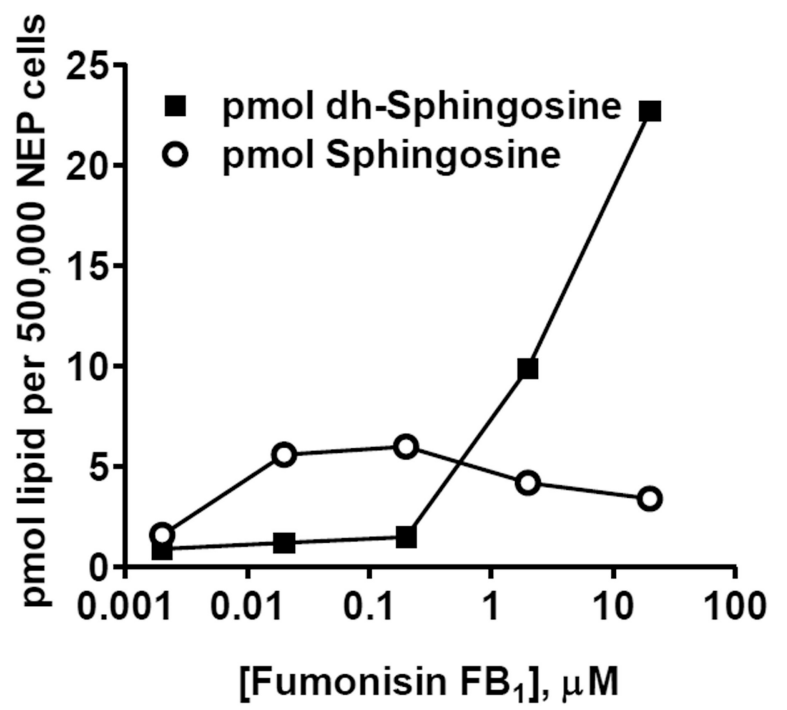

C.

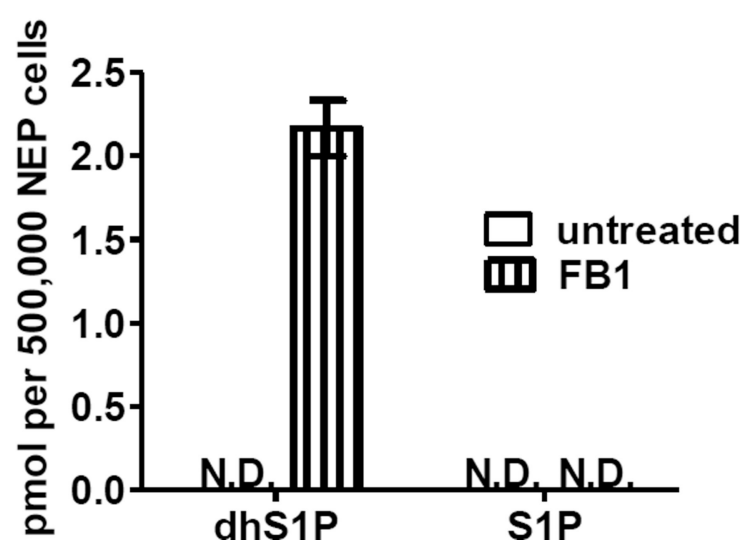

B.

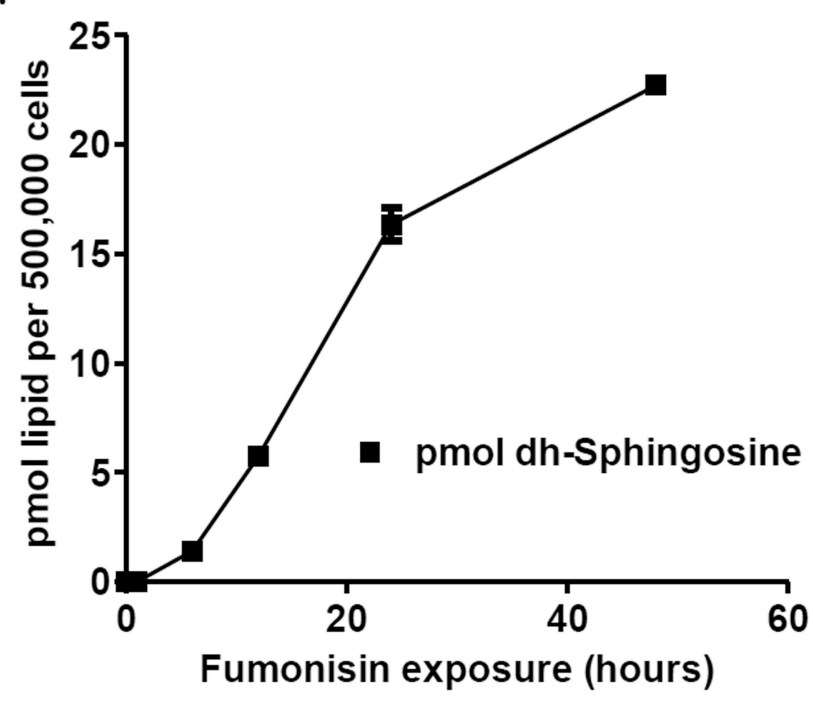

Figure 2. Analysis of sphingolipid metabolites following $\mathrm{FB}_{1}$ exposure hES-NEP cells were treated with the indicated amount of $\mathrm{FB}_{1}$. Cells were harvested and sphingolipid metabolites were quantified using LC-MS as described in Methods. Results are reported as pmol of metabolite per cells. (A) dh-sphingosine and sphingosine accumulation was determined after incubation with various concentrations of $\mathrm{FB}_{1}$ for 48 hours. (B) dhsphingosine accumulation was determined after incubation with $20 \mu \mathrm{M} \mathrm{FB}$ for various time-points. (C) dhS1P and S1P accumulation was determined after incubation with $20 \mu \mathrm{M}$ $\mathrm{FB}_{1}$ for 48 hours. (N.D. = not detected). 


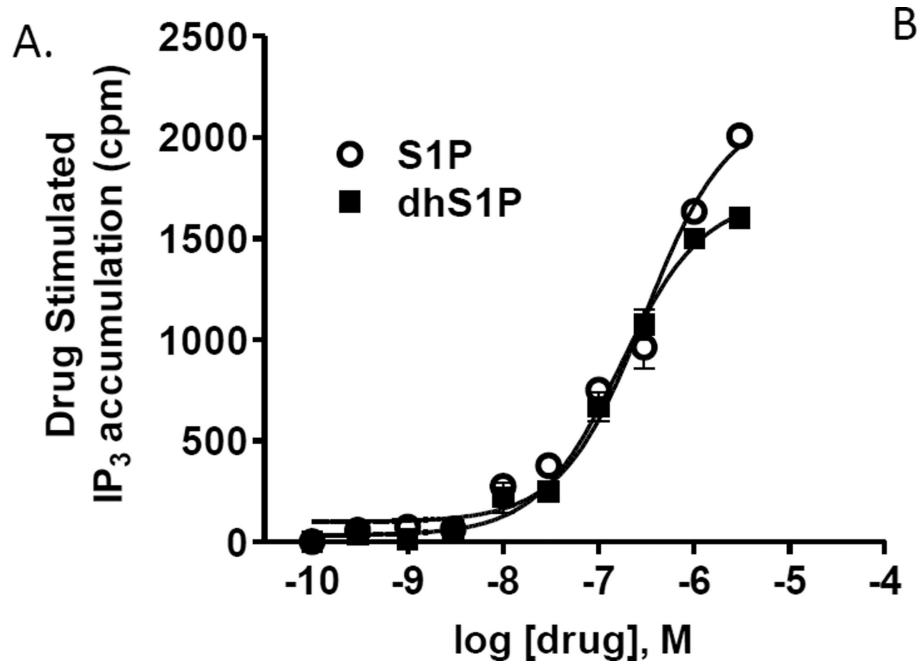

B.

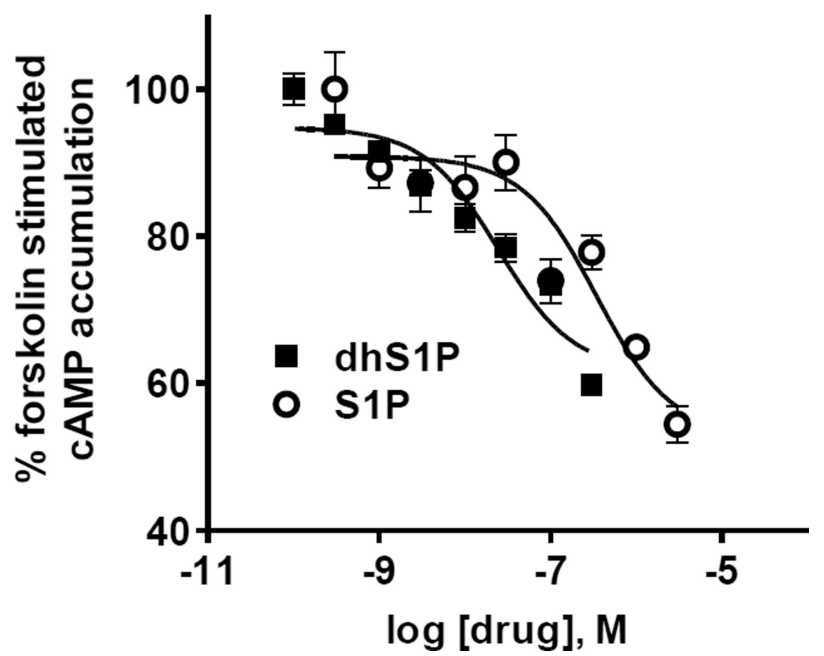

Figure 3. Pharmacology of S1P and dhS1P in hES-NEP cells

(A) hES-NEP cells were treated with S1P or dhS1P for 30 minutes and assayed for IP levels as described in Methods. Results are reported as percent of basal inositol phosphate accumulation (CPM) with counts for drug treated wells divided by values for vehicle treated wells. (B) hES-NEP cells were treated with $50 \mu \mathrm{M}$ forskolin and S1P or dhS1P for 20 minutes then assayed for cAMP levels as described in Methods. Results are reported as percent of forskolin stimulated cAMP accumulation with forskolin, +vehicle treated well representing $100 \%$. 
a
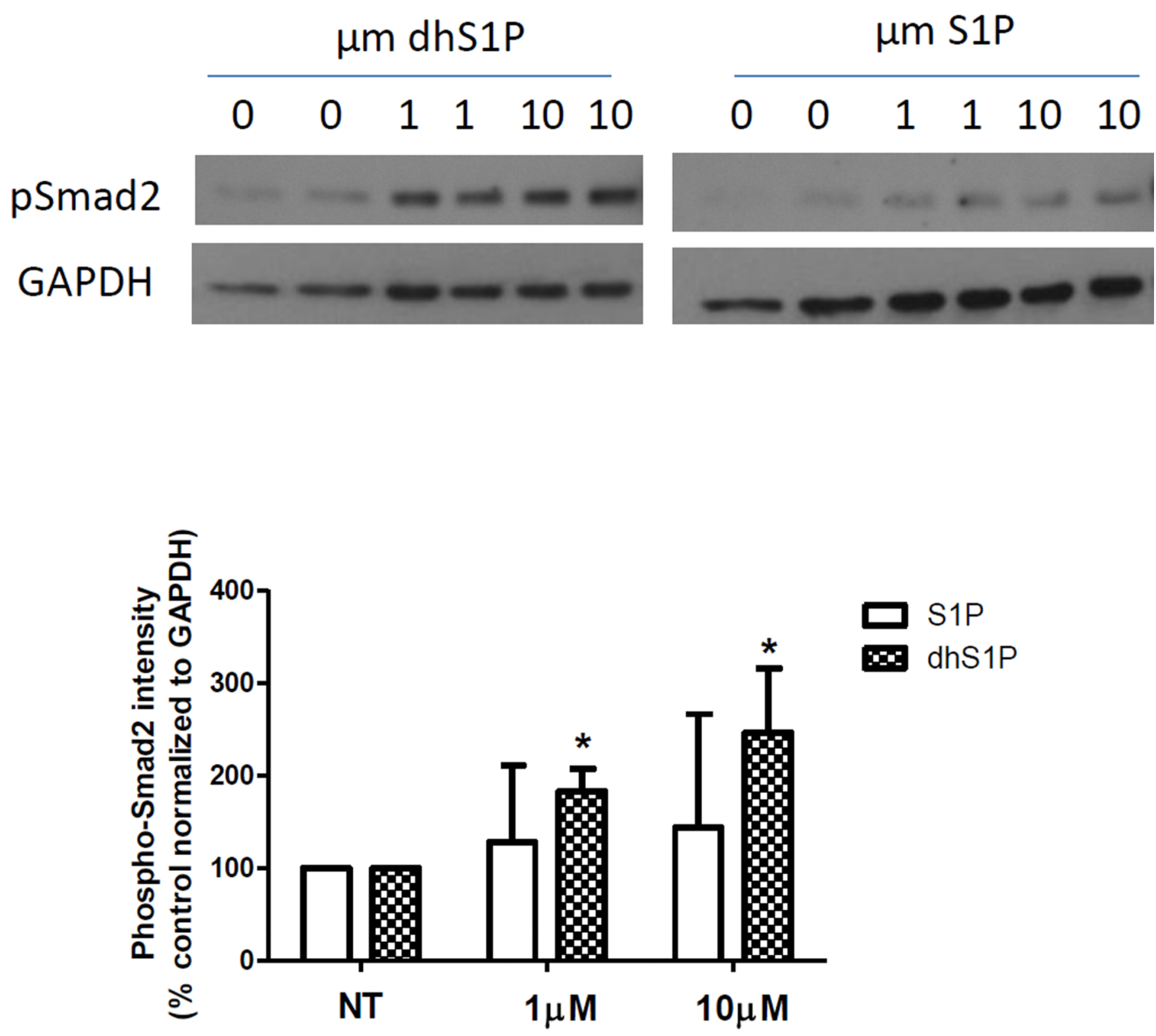
b
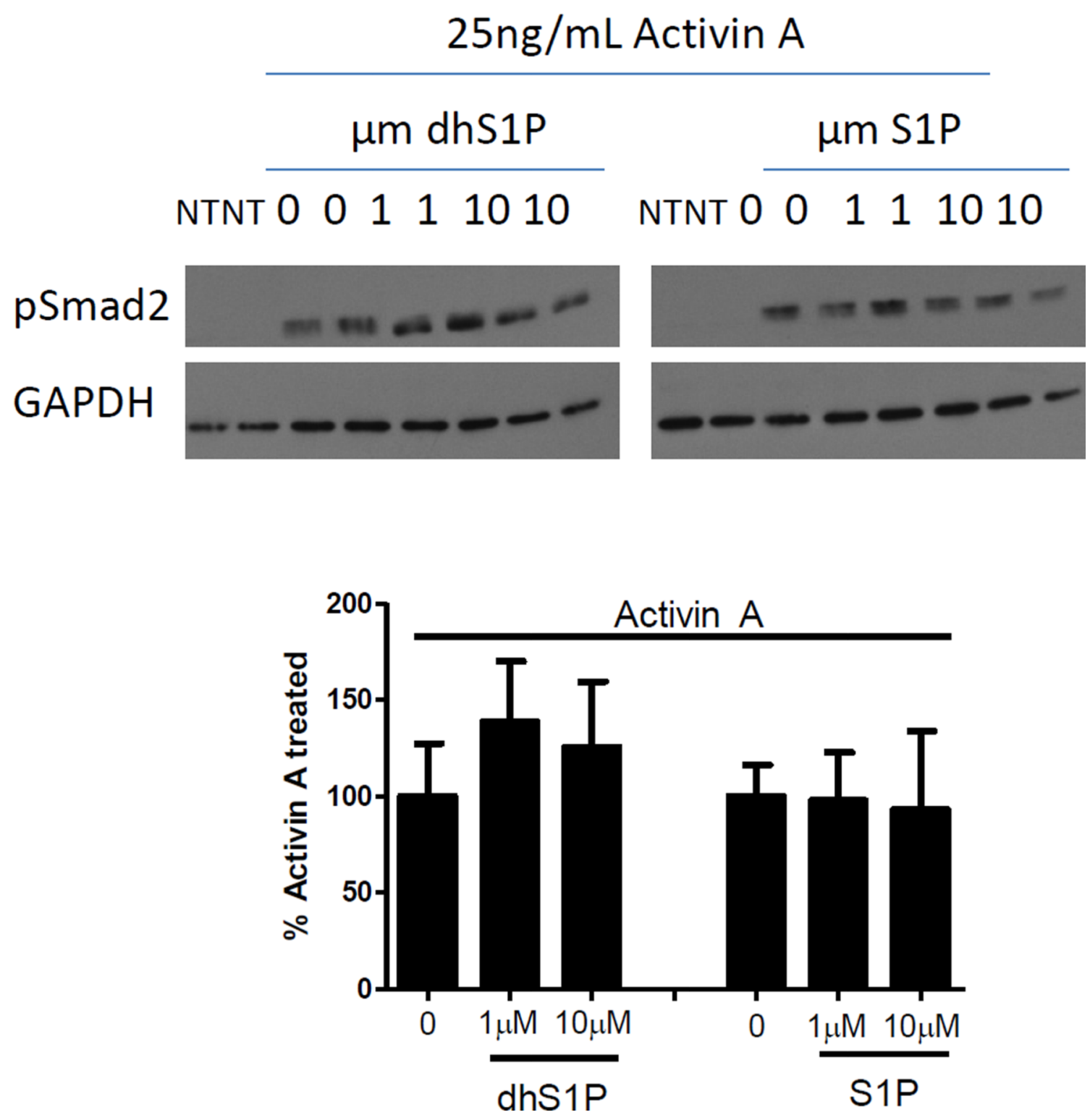

Figure 4. Role of S1P and dhS1P in Smad2 phosphorylation

(A) hES-NEP cells were untreated (NT) or treated with S1P or dhS1P for 45 minutes and then assayed for phosphorylated Smad2 as described in Methods. (B) hES-NEP cells were also treated with $25 \mathrm{ng} / \mathrm{mL}$ Activin A for 45 minutes in the presence or absence of S1P or $\mathrm{dhS} 1 \mathrm{P}$. Densitometry results are reported as the ratio of p-Smad2 to GAPDH. 
a
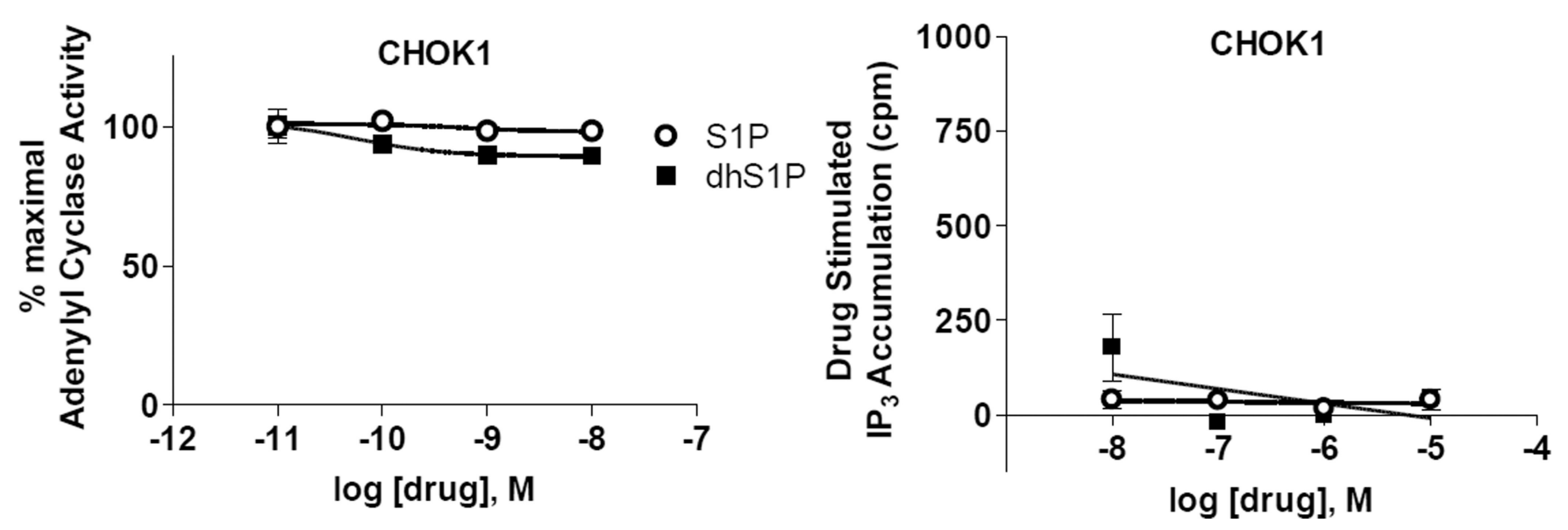
b
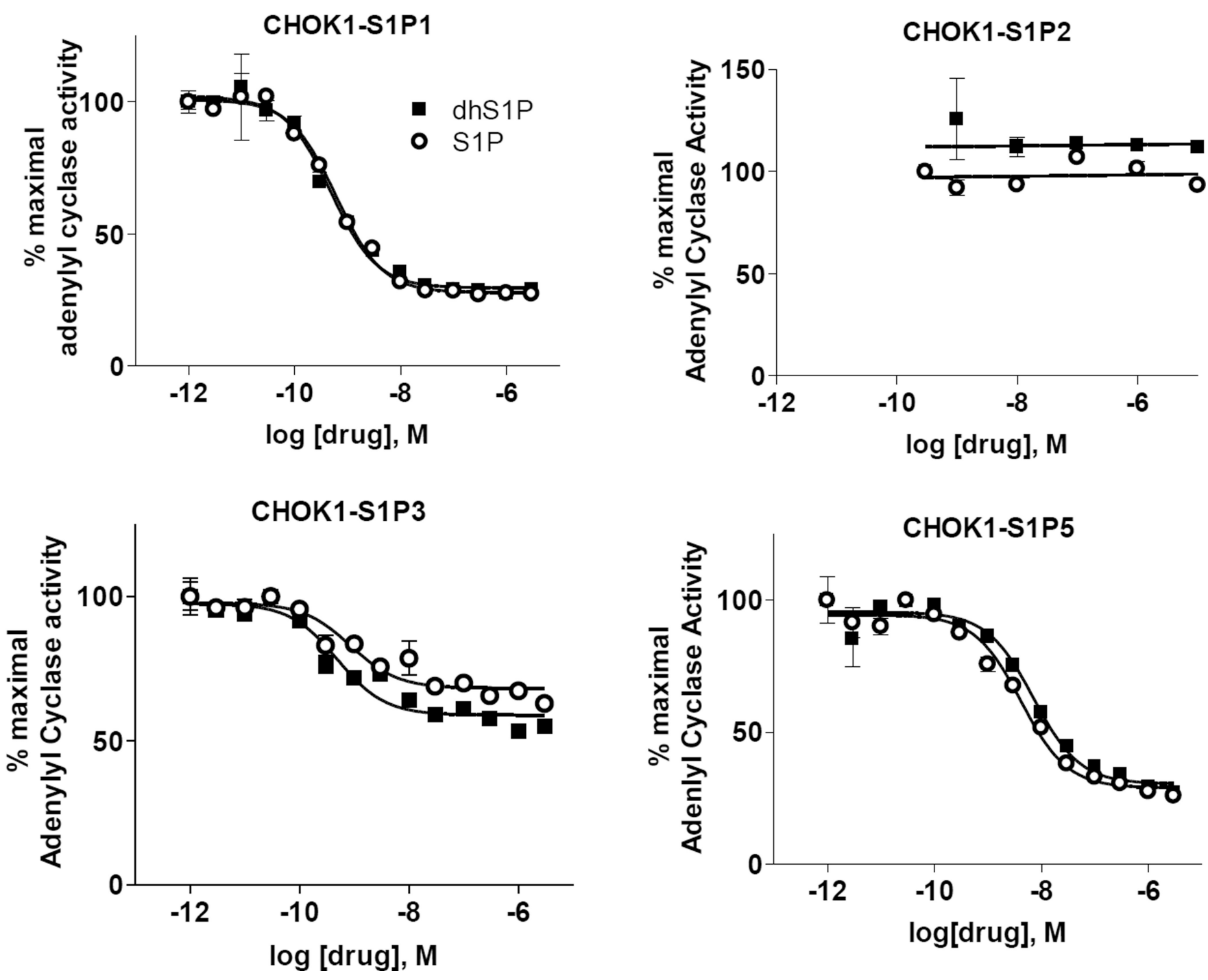
C
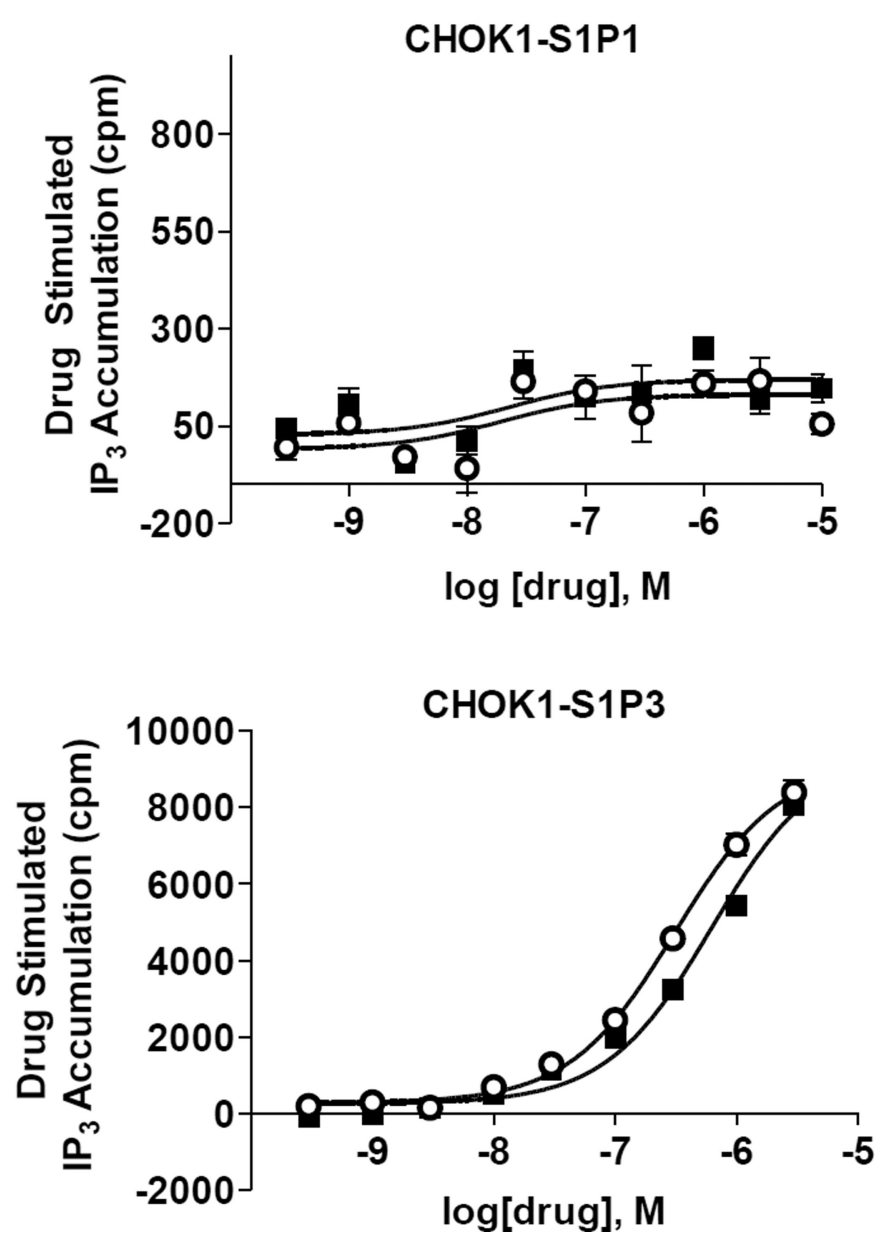
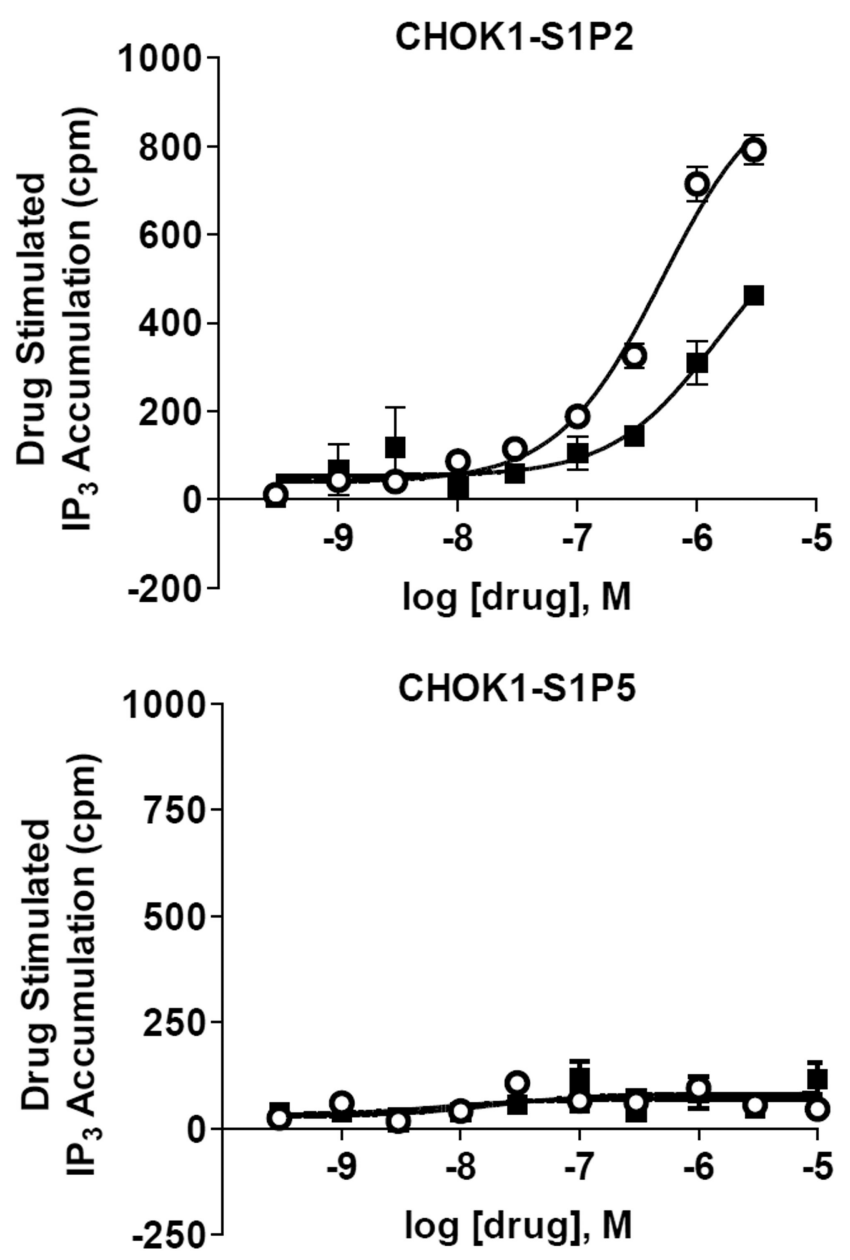
d

\section{CHOK1-S1P4}

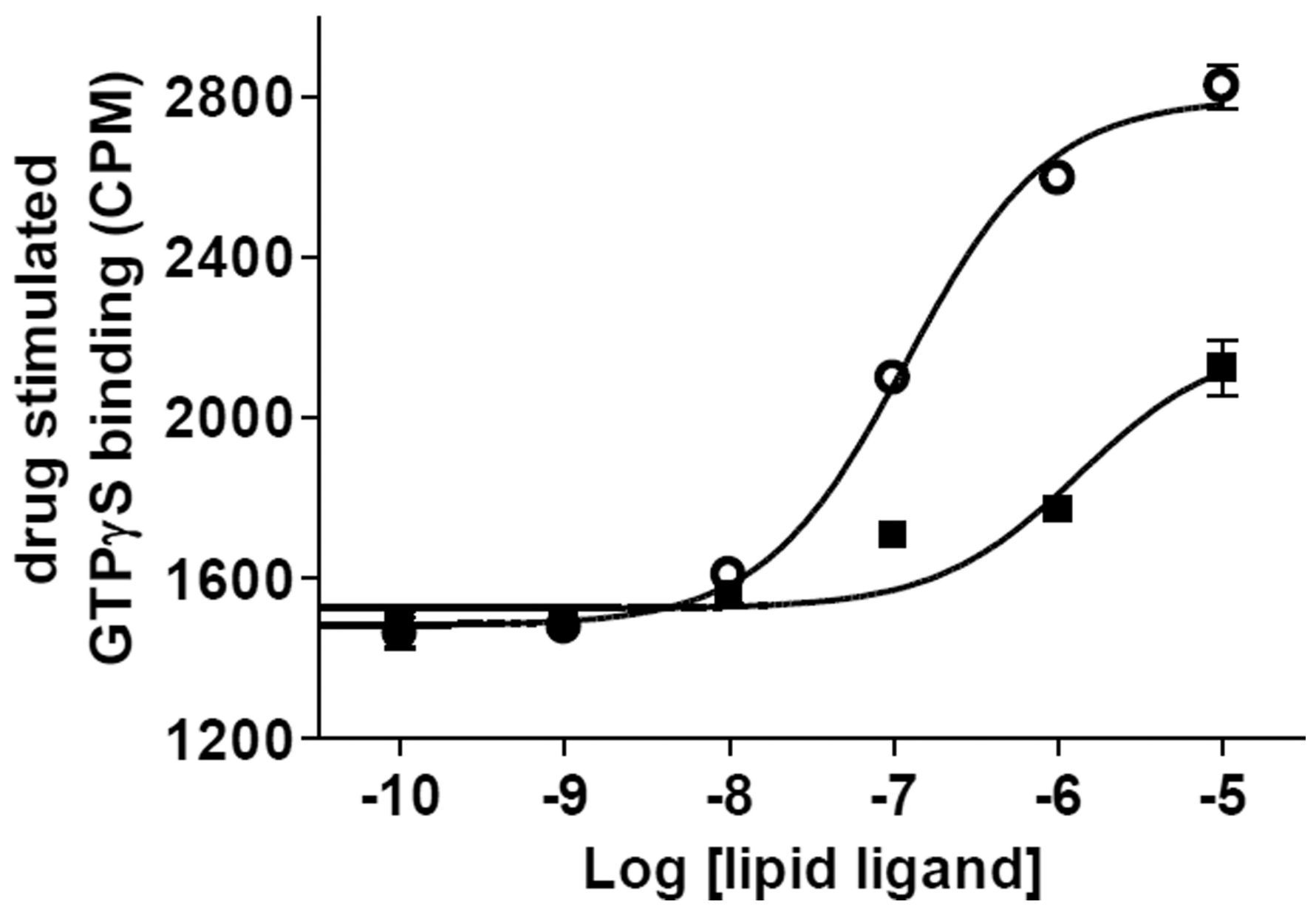

Figure 5. Pharmacology of S1P and dhS1P stimulated signaling in stable S1P receptor expressing CHO-K1 cell lines

CHO-K1 cells stably expressing individual human S1P receptors were generated as described in Methods. (A) Parental CHO-K1 cells were assayed for S1P- or dhS1Pstimulated inhibition of cAMP and activation of PLC as described. (B) Stable receptorexpressing $\mathrm{CHO}-\mathrm{K} 1$ cells were treated with $50 \mu \mathrm{M}$ forskolin and the indicated drugs for 20 minutes then assayed for cAMP levels as described in Methods. Results are reported as percent of forskolin stimulated cAMP accumulation with forskolin, + vehicle treated well representing 100\%. (C) Stable receptor-expressing CHO-K1 cells were treated with each of the indicated drugs for 30 minutes and assayed for IP levels as described in Methods. Results are reported as percent of basal inositol phosphate accumulation (CPM) with counts for drug treated wells divided by values for vehicle treated wells. (D) GTP $\gamma \mathrm{S}$ binding assay using membranes from CHO-K1 S1P4 cells. 

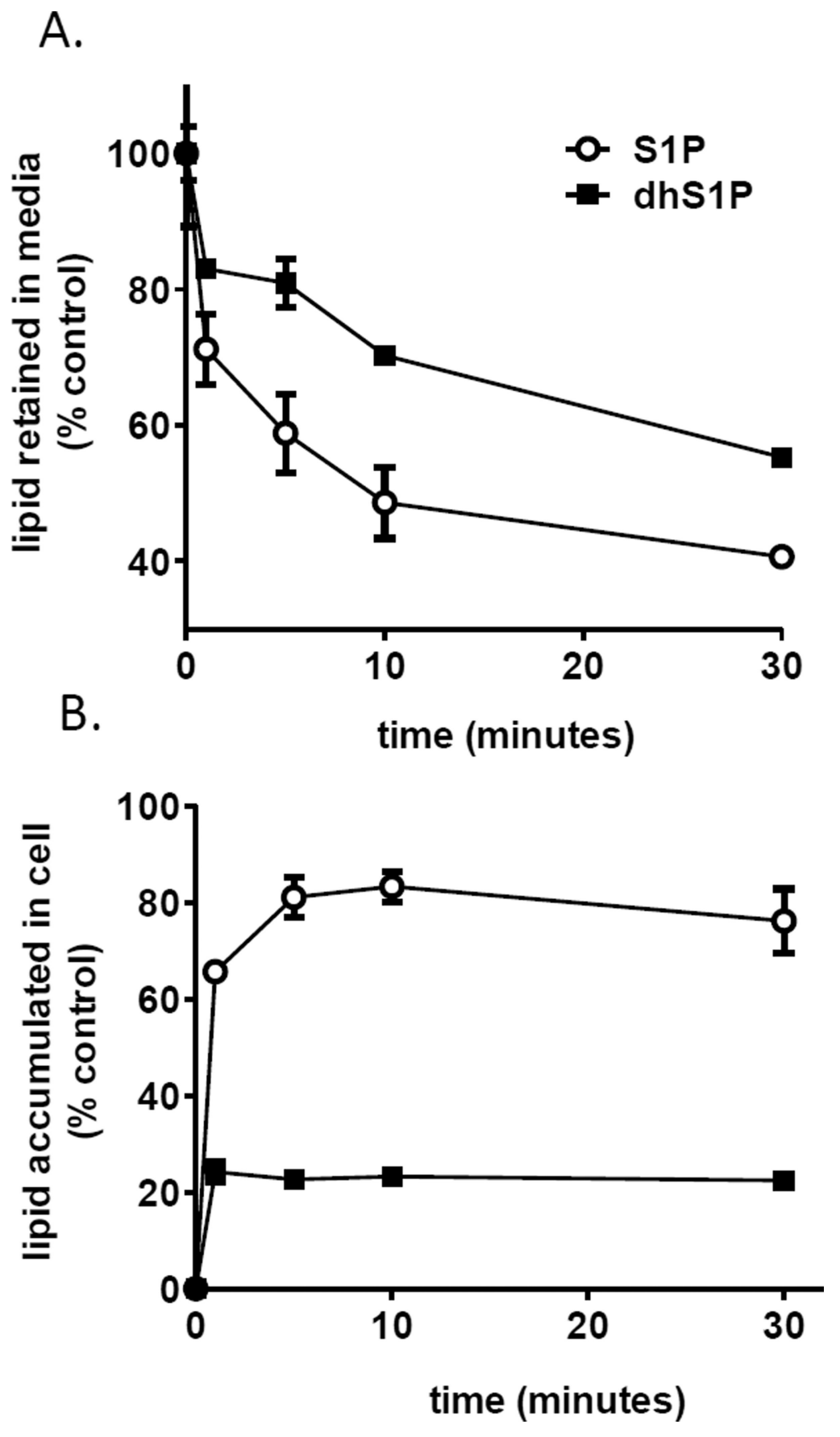

Figure 6. Analysis of S1P and dhS1P in hES-NEP media and cells hES-NEP cells ( $~ 500,000$ per well) were untreated (NT) or treated with $1 \mu \mathrm{M} \mathrm{S1P}$ or dhS1P for 2, 5, 10, and 30 minutes. Cells and media were harvested and sphingolipid metabolites were quantified using LC-MS as described in Methods. Results are reported as \% of total exogenous applied lipid. (A) S1P and dhS1P in hES-NEP media. (B) S1P and dhS1P in hES-NEP cells. 